\title{
Avaliação da substituição da silagem de milho por volumoso extrusado na dieta de ovinos
}

\author{
Débora Adriana de Paula Silva ${ }^{1}$; Gustavo Roberto Dias Rodrigues ${ }^{2}$; Marco Túlio Santos Siqueira ${ }^{3}$; Erica Beatriz \\ Schultz $^{4 *}$; Karla Alves Oliveira ${ }^{5}$; Gilberto de Lima Macedo Júnior ${ }^{6}$
}

DOI: https://doi.org/10.35699/2447-6218.2021.32930

\begin{abstract}
Resumo
Objetivou-se avaliar os efeitos da substituição da silagem de milho por volumoso extrusado (Urochloa brizantha $c v$ Marandu) sobre o consumo e digestibilidade da matéria seca, comportamento ingestivo, curva glicêmica e metabólitos sanguíneos de ovinos. Foram utilizadas 20 ovelhas da raça Santa Inês com idade superior a três anos e peso corporal médio de $54,5 \mathrm{~kg}$, distribuídas em delineamento inteiramente casualizado, com quatro tratamentos e cinco repetições. Os tratamentos consistiram na inclusão de volumoso comercial extrusado, Foragge ${ }^{\circledR} 70 \%(\mathrm{~F})\left(\mathrm{Nuttrata}{ }^{\circledR}\right)$ em substituição à silagem de milho (S) nas proporções: 20\%F:80\%S; 40\%F:60\%S; 60\%F e 40\%S e 80\%F:20\%S. Foi realizada a análise de regressão a 5\% de significância. À medida que aumentou a inclusão de volumoso extrusado houve aumento no consumo de matéria seca (CMS), CMS em relação ao peso corporal, CMS em relação ao peso metabólico, peso de fezes na matéria natural e matéria seca $(\mathrm{P}<0,05)$. Houve aumento linear para eficiência de ingestão, eficiência de ruminação, eficiência de mastigação, ureia e glicemia $(\mathrm{P}<0,05)$. A digestibilidade da matéria seca, e os metabolitos energéticos não foi alterada $(\mathrm{P}>0,05)$. O tempo de ruminação obteve resposta quadrática quanto a substituição da silagem de milho pelo volumoso extrusado $(\mathrm{P}<0,05)$. Conclui-se que a substituição da silagem em até $80 \%$ pelo volumo extrusado é recomendada, por melhorar o consumo sem efeitos deletérios na digestibilidade e metabólitos sanguíneos.
\end{abstract}

Palavras chave: Extrusão. Ovis aries. Ruminantes. Urochloa brizantha.

\section{Evaluation replacement of corn silage by extruded roughage on sheep diet}

\begin{abstract}
:
The aim was to evaluate the effects of replacing corn silage with extruded roughage (Urochloa brizantha cv Marandu) on dry matter intake and digestibility, ingestive behavior, glycemic curve and blood metabolites of sheep. Twenty Santa Inês ewes, with more three years old and average body weight of $54.5 \mathrm{~kg}$ were used, assign in a completely randomized design, with four treatments and five repetitions. The treatments were use the extruded commercial roughage, Foragge ${ }^{\circledR} 70 \%$ (F) $\left(\right.$ Nuttrata $^{\circledR}$ ) to replace corn silage (S) in the proportions: $20 \% \mathrm{~F}: 80 \% \mathrm{~S} ; 40 \% \mathrm{~F}: 60 \% \mathrm{~S}$; $60 \% \mathrm{~F}$ and $40 \% \mathrm{~S}$ and $80 \% \mathrm{~F}: 20 \% \mathrm{~S}$. Regression analysis was performed at 5\% significance level. As the inclusion of extruded roughage increased, there was an increase in the dry matter intake (DMI), DMI relation to body weight, DMI relation to metabolic weight, weight of feces in natural and dry matter. There was linear increase to efficiency of
\end{abstract}

${ }^{1}$ Universidade Federal de Uberlândia. Uberlândia, MG. Brasil.

https://orcid.org/0000-0003-3052-0544

${ }^{2}$ Universidade Federal de Uberlândia. Uberlândia, MG. Brasil.

https://orcid.org/0000-0001-9438-3724

${ }^{3}$ Universidade Federal de Uberlândia. Uberlândia, MG. Brasil.

https://orcid.org/0000-0002-2098-8568

${ }^{4}$ Universidade Federal de Viçosa. Viçosa, MG. Brasil.

http://orcid.org/0000-0003-1916-2117

${ }^{5}$ Universidade Estadual Paulista. Jaboticabal, SP. Brasil.

https://orcid.org/0000-0002-7792-2615

${ }^{6}$ Universidade Federal de Uberlândia. Uberlândia, MG. Brasil. https://orcid.org/0000-0001-5781-7917

*Autor para correspondência: ericabeatrizschultz@gmail.com

Recebido para publicação em 03 de Abril de 2021. Aceito para publicação 25 de Julho de 2021

e-ISSN: 2447-6218 / ISSN: 2447-6218. Atribuição CC BY. 
Silva, D. A. de P. et al.

eating, rumination and chewing, urea and blood glucose $(\mathrm{P}<0.05)$. The dry matter digestibility and energy metabolites was not altered $(\mathrm{P}>0.05)$. The rumination time showed a quadratic response regarding the replacement of corn silage by extruded roughage $(\mathrm{P}<0.05)$. It is concluded that the replacement of silage in up to $80 \%$ by the extruded volume is recommended, as it improves intake without deleterious effects on digestibility and blood metabolites.

Key words: Extrusion. Ovis aries. Ruminants. Urochloa brizantha.

\section{Introdução}

A alimentação dos animais ruminantes é predominantemente composta por alimentos volumosos em pastagens. Entretanto, no Brasil, o clima tropical influencia diretamente em duas estações definidas: água e seca, sendo a última responsável por reduzir a qualidade e produtividade das pastagens nessa época do ano. Sendo assim, no intuito de evitar déficits produtivos, a prática de ensilagem é amplamente realizada na finalidade de garantir ofertas qualitativas e quantitativas alimentares ao requerimento animal e ao mesmo tempo aumentar a eficiência da utilização das pastagens (Macêdo et al., 2019).

A silagem de milho é a ensilagem de maior expressão no Brasil, uma vez que esse alimento produz boa quantidade de matéria seca por área, fornecendo energia e proteína, além da aceitabilidade pelos animais ruminantes (Mourão et al., 2012). Em contrapartida, para a obtenção de uma silagem de qualidade, verifica-se a necessidade de seguir rigorosamente os processos de ensilagem, como, implementação da cultura, tratos culturais, fertilidade do solo, composição químico-bromatológica, estágio de maturação da forrageira, manejo de colheita, grau de processamento do material, higiene de silo, tipo, tempo de fechamento, compactação e vedação do silo (Macêdo et al., 2019). Todas essas práticas refletem na qualidade da silagem que será obtida, sendo fundamentais para que o alimento fornecido aos animais esteja com qualidade nutricional próxima à forrageira de origem, visto que os aspectos nutritivos da silagem estão amplamente ligados à composição e digestibilidade da forrageira de origem.

Com isso, no intuito de auxiliar o manejo e diminuir possíveis riscos de oscilação na qualidade dos alimentos volumosos fornecidos aos animais, atualmente, novos métodos de conservação de alimentos vêm sendo inseridos no mercado, como é o caso dos volumosos extrusados. De acordo com Reddy \& Reddy (2015), no processo de extrusão os alimentos passam pela combinação entre calor, pressão e trabalho mecânico, até a passagem por um cilindro, onde no final deste há a presença de uma matriz com furos de menor diâmetro, formando o produto extrusado final. Como a temperatura dessa atividade ultrapassa $100^{\circ} \mathrm{C}$, a extrusão tem a capacidade de promover o rompimento das células vegetais, o que proporciona a total fusão entre os ingredientes utilizados.

A utilização de produtos extrusados na nutrição animal gera benefícios ao sincronismo das fontes ali- mentares, uma vez que as proteínas, energia, minerais, aditivos e fibras tornam-se um único alimento e com isso, a fermentação ruminal é ampliada e resulta em melhor aproveitamento dos alimentos pelos animais, como demonstrado por Oliveira et al. (2019) e Silva et al. (2020). Além disso, o ambiente ruminal é beneficiado, uma vez que os ingredientes serão liberados em equilíbrio no rúmen de forma sincronizada, contribuindo para manutenção do $\mathrm{pH}$ ruminal sem a necessidade de tamponantes. Por fim, a digestibilidade dos alimentos também é aumentada, uma vez que a extrusão quebra a fibra das paredes celulares (Reddy \& Reddy, 2015).

Portanto nossa hipótese é que a substituição da silagem de milho por volumoso extrusado pode melhorar a dinâmica ruminal e o aproveitamento da dieta para ovinos. Objetivou-se avaliar níveis de substituição da silagem de milho por volumoso extrusado (Urochloa brizantha cv Marandu) sobre o consumo e digestibilidade da matéria seca, comportamento ingestivo e metabólitos sanguíneos de ovinos.

\section{Material e Métodos}

O ensaio experimental foi realizado no setor de caprinos e ovinos presente na fazenda Capim Branco, da Universidade Federal de Uberlândia (UFU), durante o mês de julho de 2017 com duração total de 21 dias. Os quinze primeiros dias do experimento foram destinados à adaptação dos animais a dieta experimental, e os últimos seis dias para mensuração e coleta de fezes, urina, sangue, água, amostras de sobras e ofertado de alimento. Todos os manejos utilizados contaram com a aprovação da Comissão de Ética e Utilização dos Animais (CEUA) da UFU sob o número de protocolo 092/16.

Foram utilizadas vinte ovelhas da raça Santa Inês, não gestantes e não lactantes, com peso corporal médio de 54,5 kg e idade média de três anos. Todos os animais foram pesados e vermifugados com Levamisol (via oral) no primeiro dia do experimento e feita observação da mucosa ocular. Posteriormente, em um galpão de alvenaria, os animais ficaram alocados em gaiolas metabólicas individuais equipadas com comedouro, bebedouro, saleiro, piso ripado e artefato de separação de fezes e urina de acordo com padrão dos Institutos Nacionais de Ciência e Tecnologia (INCT). 
Avaliação da substituição da silagem de milho por volumoso extrusado na dieta de ovinos

Os tratamentos foram compostos por níveis de inclusão de volumoso comercial extrusado, Foragge ${ }^{\circledR}$ 70\% (F) (Nuttrata Nutrição Animal, Itumbiara - GO, Brasil) em substituição a silagem de milho (S) nas proporções: $20 \%$ de Foragge $^{\circledR}$ e $80 \%$ de Silagem de milho (20\%F:80\%S); 40\% de Foragge ${ }^{\circledR}$ e $60 \%$ de Silagem de milho (40\%F:60\%S); 60\% de Foragge ${ }^{\circledR}$ e 40\% de Silagem de milho $\left(60 \%\right.$ F e $40 \%$ S) e $80 \%$ de Foragge ${ }^{\circledR}$ e $20 \%$ de Silagem de milho (80\%F:20\%S). O fornecimento da alimentação ocorreu duas vezes ao dia, às 08:00hrs e posteriormente às $16: 00 \mathrm{hrs}$, onde foi ofertado $50 \%$ do total diário em cada um desses turnos. Além disso, a dieta experimental foi balanceada de modo com que houvesse sobras entre 5-10\% do total fornecido. Durante todos os 21 dias do experimento os animais tiveram livre acesso à água e sal mineral ad libitum.

O Foragge ${ }^{\circledR} 70 \%$ é um produto de forma extrusada, composto por 70\% de capim da espécie Urachloa brizantha, cultivar Marandu, amido, farelo de girassol e premix vitamínico e mineral. A composição química do Foragge ${ }^{\circledR} 70 \%$, da silagem de milho e das dietas estão descritos na Tabela 1.

Tabela 1 - Composição química dos ingredientes e das dietas experimentais

\begin{tabular}{|c|c|c|c|c|c|c|c|}
\hline \multirow[b]{2}{*}{ Item } & \multicolumn{7}{|c|}{ Nutrientes (\%) } \\
\hline & MS & PB & FDN & FDA & NDT & CINZAS & AMIDO \\
\hline Foragge ${ }^{\circledR} 70 \% *$ & 90 & 6,68 & 64,44 & 35,7 & 64,44 & 4,6 & 24,3 \\
\hline Silagem de milho*** & 28,02 & 8 & 57,11 & 36,17 & 62,52 & 10,3 & $23,63^{* * *}$ \\
\hline \multicolumn{3}{|c|}{ Tratamentos } & MS & PB & FDN & FDA & NDT \\
\hline \multicolumn{3}{|c|}{$20 \%$ Foragge $^{\circledR}: 80 \%$ Silagem de milho } & 43,26 & 7,93 & 56,4 & 36,07 & 62,9 \\
\hline \multicolumn{3}{|c|}{$40 \%$ Foragge ${ }^{\circledR}: 60 \%$ Silagem de milho } & 55,32 & 7,86 & 55,7 & 35,98 & 63,3 \\
\hline \multicolumn{3}{|c|}{$60 \%$ Foragge ${ }^{\circledR}: 40 \%$ Silagem de milho } & 65,33 & 7,79 & 54,99 & 35,88 & 63,6 \\
\hline \multicolumn{3}{|c|}{ 80\% Foragge ${ }^{\circledR}: 20 \%$ Silagem de milho } & 76,98 & 7,72 & 54,29 & 35,79 & 64 \\
\hline
\end{tabular}

MS: matéria seca; PB: proteína bruta; FDN: fibra em detergente neutro; FDA: fibra em detergente ácido; NDT: nutrientes digestíveis totais; *Valores fornecidos pelo fabricante Nutratta (C).*Valores obtidos através de análises bromatológicas efetuadas no Laboratório de Nutrição Animal da Universidade Federal de Uberlândia. ***Dado retirado das Tabelas Brasileiras de Composição de Alimentos para Bovinos, por Valadares Filho et al., (2018).

Em todos os dias de coleta, os alimentos ofertados, sobras e fezes foram pesados e amostrados utilizando balança eletrônica com precisão de cinco gramas. Foram feitas amostras compostas provenientes de cada animal para realização das análises químicas e cálculo de consumo e digestibilidade da matéria seca. No final do período de coleta, estas amostras foram armazenadas em freezers horizontais à $-15^{\circ} \mathrm{C}$, para conservação, e em seguida os alimentos ofertados, sobras e fezes foram pré secos em estufa ventilada à $55^{\circ} \mathrm{C}$ durante 72 horas, até ser obtido peso constante. Em seguida, foram trituradas com a utilização de um moinho de facas tipo Willey, em partículas de $1 \mathrm{~mm}$. Após esse procedimento, as amostras foram levadas ao Laboratório de Nutrição Animal da UFU, onde foi verificada a matéria seca das porções de ofertado, sobras e fezes, em estufa à $105^{\circ} \mathrm{C}$ durante 24 horas.

A partir do cálculo da matéria seca foram calculados o consumo de matéria seca (CMS) e a digestibilidade da matéria seca através da equação proposta por Maynard et al., (1984).
A urina foi amostrada por meio de baldes com telas para a retenção das fezes, que foram recolhidas em bandejas plásticas. O volume de urina foi mensurado com a utilização de provetas graduadas de plástico com capacidade de dois litros e precisão de $20 \mathrm{~mL}$. Foram quantificados o volume urinário excretado por cada animal durante o período de 24 horas.

A densidade da urina foi avaliada utilizando um refratômetro manual portátil Megabrix ${ }^{\circledR}$ (Fremont, Ohio, Estados Unidos) com o auxílio de pipetas descartáveis, onde $1 \mathrm{~mL}$ de urina foi transferido do balde coletor para o prisma do optômetro. Esse procedimento foi realizado sob luz fluorescente, sempre na mesma posição. Entre a mensuração de cada amostra, o refratômetro foi higienizado e seco com papel toalha no intuito de não ocorrer interferências entre os resultados.

O escore fecal foi mensurado pela escala proposta por Gomes et al. (2012), onde na escala um (1) as fezes são ressecadas e sem brilho; na escala dois (2) as fezes são normais; na escala três (3) as fezes são ligeiramente amolecidas; na escala quatro 
Silva, D. A. de P. et al.

(4) as fezes são amolecidas, perdendo o formato e coladas umas às outras; na escala cinco (5) as fezes são amolecidas e sem formato normal; e na escala seis (6) as fezes são diarreicas.

A ingestão de água pelos animais foi calculada baseado na diferença entre a quantidade de água ofertada diariamente e as sobras verificadas no dia seguinte, sendo levado em consideração a quantidade de água que evaporava. A mensuração da água oferecida foi realizada com a utilização de uma proveta graduada de plástico com capacidade de dois litros e exatidão de $20 \mathrm{~mL}$. Diariamente eram oferecidos seis litros de água para cada animal e caso houvesse necessidade, fornecia-se quantidades maiores. A evaporação foi medida a cada 24 horas por meio da inserção de um balde no galpão experimental contendo seis litros de água, onde o mesmo ficou em local de acesso restrito aos animais. O balde de evaporação foi colocado durante o momento de fornecimento de água, em uma superfície plana correspondente à altura dos baldes inseridos nas gaiolas metabólicas. A mensuração da quantidade evaporada também foi por meio da diferença entre os seis litros ofertados e as sobras verificadas no dia seguinte. A quantidade total de água evaporada foi descontada do consumo diário de água de cada animal. O cálculo do consumo de água total foi feito somando a água do balde com a água contida no alimento ingerido.

No quinto dia do período de coleta, ocorreu a mensuração do comportamento ingestivo, onde os animais foram submetidos à observação visual por pessoas treinadas, em sistema de revezamento, dispostas de maneira a não incomodar os animais durante vinte e quatro horas, uma vez ao longo do período de coleta. No período noturno, o ambiente recebeu iluminação artificial e as luzes foram mantidas acessas durante cinco dias antes da avaliação para promover a adaptação dos animais às mesmas. Os animais foram avaliados a cada cinco minutos por 24 horas, sendo observado a ingestão de alimento ou água separadamente (ING), ruminação (RUM) ou ócio de acordo com a metodologia proposta por Fischer et al. (1998).

Os cálculos das atividades foram feitos em minutos por dia, admitindo que, nos cinco minutos subsequentes a cada observação, o animal permaneceu na mesma atividade. Já o tempo total gasto em mastigação (MAST) foi determinado somando-se os tempos gastos em ingestão (ING) e ruminação (RUM). As eficiências de ingestão (Eing), mastigação
(Emast) e ruminação (Erum) foram obtidas segundo Polli et al. (1996), dividindo o consumo de matéria seca por cada atividade dispendida.

As coletas de sangue para avaliação dos componentes bioquímicos foram realizadas no primeiro, terceiro e quinto dia de coleta do experimento, antes da primeira refeição ofertada no dia. As colheitas de sangue ocorreram por venopunção da jugular com tubos Vacutainer ${ }^{\circledR}$ sem anticoagulante. Os componentes bioquímicos avaliados para determinação do metabolismo energético foram: triglicerídeos, colesterol e frutosamina; e para determinação do metabolismo proteico foram: ureia, proteínas totais, ácido úrico, albumina e creatinina.

Já para a avaliação glicêmica, as colheitas foram realizadas no último dia de coleta de dados, às 8:00 (antes da primeira refeição), 11:00, 14:00, 17:00 e 20:00 horas. Excepcionalmente nesse dia, a segunda refeição somente foi ofertada após o recolhimento das 20:00 horas. Essas amostras foram coletadas por meio de venopunção da jugular com a utilização de tubos Vacutainer ${ }^{\circledR}$ (BD) contendo fluoreto e EDTA, identificados para cada animal. Não foram mensurados os efeitos do estresse da coleta a cada três horas nos animais.

Todas as amostras de sangue coletadas foram centrifugadas a 3000 rotações por minuto, durante 10 minutos. Após a separação do soro em alíquotas, estes ficaram armazenados em freezer à $-15^{\circ} \mathrm{C}$ para futuras análises laboratoriais. As amostras foram processadas em analisador bioquímico automatizado Bioplus ${ }^{\circledR} 2000$ (Bioplus Produtos para Laboratório Ltda, Barueri - SP, Brasil), usando kit comercial (Lab Test Diagnósticos S. A. ${ }^{\circledR}$, Lagoa Santa - MG, Brasil)

Para a maioria das variáveis analisadas utilizou-se o delineamento inteiramente casualizado com quatro tratamentos e cinco repetições. Já para glicemia considerou-se delineamento inteiramente ao acaso com medidas repetidas no tempo. Esta variável foi testada para condição de esfericidade, que não foi aceita. Portanto, utilizou-se a análise de modelos mistos, em que foram avaliadas todas as estruturas de covariâncias (S) disponíveis no pacote do software SAS (SAS Institute, 2012) que modelam a dependência dos erros do modelo. Para selecionar a estrutura de covariância que melhor explique a correlação residual, foi utilizado o critério de informação de Akaike (AIC), sendo escolhida, para cada variável, a estrutura que resultou no menor valor de AIC após a análise (Silva et al., 2015).

Todos os dados foram testados quanto a normalidade e homocedasticidade das variâncias dos tratamentos. As variáveis normais e com variâncias 
Avaliação da substituição da silagem de milho por volumoso extrusado na dieta de ovinos

homogêneas foram submetidas a análise de regressão, considerando os efeitos linear e quadrático para a probabilidade de erro tipo I a 5 \% de significância. Os dados referentes ao escore fecal foram analisados por estatística não paramétrica, através do teste de Kruskal e Wallis (1952) seguido pelo procedimento de Conover (1980) a 5 \% de significância.

\section{Resultados e discussão}

À medida que aumentou a proporção de volumoso extrusado na dieta: o consumo de matéria seca (CMS), CMS em relação ao peso corporal (CMS/PC) e CMS pelo peso metabólico (CMS/PC ${ }^{0,75}$ ) aumentaram linearmente $(\mathrm{P}<0,05)$ (Tabela 2). De acordo com Zereu (2016), dentre os fatores que podem influenciar no consumo dos animais, estão a composição física e química, tamanho das partículas e balanço dos nutrientes dos alimentos ofertados. $\mathrm{O}$ uso alimento extrusado contribuiu para o aumento no consumo. Isto pois, o uso de volumoso extrusado em comparação a silagem de milho aumentou o consumo devido ao processamento dos alimentos durante o processo de extrusão, como gelatinização do amido e desnaturação das proteínas (Oliveira et al., 2018).

Tabela 2 - Consumo e digestibilidade da matéria seca em ovelhas consumindo níveis crescentes de Foragge ${ }^{\circledR} 70 \%$ em relação à silagem de milho

\begin{tabular}{|c|c|c|c|c|c|c|c|}
\hline & \multicolumn{4}{|c|}{ Tratamentos } & \multirow[b]{2}{*}{ P-valor } & \multirow[b]{2}{*}{ MG } & \multirow[b]{2}{*}{ CV (\%) } \\
\hline & 20F:80S & 40F:60S & 60F:40S & $80 F: 20 S$ & & & \\
\hline $\mathrm{CMS}^{1}\left(\mathrm{~kg} \cdot \mathrm{dia}^{-1}\right)$ & 1,50 & 1,84 & 2,07 & 2,37 & 0,0085 & 1,95 & 17,84 \\
\hline CMS/PC $(\%)$ & 2,34 & 2,84 & 3,34 & 3,60 & $<0,001$ & 3,00 & 8,58 \\
\hline $\mathrm{CMS} / \mathrm{PC}^{0,75}$ & 66,19 & 80,65 & 91,38 & 102,59 & $<0,001$ & 85,2 & 8,94 \\
\hline DMS (\%) & 58,95 & 55,25 & 53,19 & 60,40 & 0,2106 & 56,95 & 10,03 \\
\hline
\end{tabular}

CMS: consumo de matéria seca; PC: peso corporal; DMS: digestibilidade da matéria seca; MG: média geral; CV: coeficiente de variação; ${ }^{1} \mathrm{Y}=$ $1,244850+0,014130 x, R^{2}=99,57 \% ;{ }^{2} Y=1,962830+0,020936 x, R^{2}=99,42 \% ;{ }^{3} Y=55,222680+0,599690 x, R^{2}=99,51 \%$.

Outro fator para o aumento do consumo de matéria seca com uso de volumoso extrusado foi sobre o tamanho das partículas em comparação a silagem de milho. Como as partículas do volumoso extrusado possuem tamanho aproximado de $2 \mathrm{~mm}$ em contrapartida a $1 \mathrm{e}$ $2 \mathrm{~cm}$ da silagem de milho. Assim nos tratamentos com maior proporção de silagem de milho está permaneceu por mais tempo no ambiente ruminal, o que provocou o efeito de enchimento, e consequentemente, contribuiu para a redução no consumo (Tabela 2).

Em relação aos valores médios de consumo de matéria seca, a recomendação feita pelo NRC (2007) para a categoria animal avaliada é de $1,05 \mathrm{~kg} /$ dia de CMS. Sendo assim, todos os tratamentos verificados apresentaram-se superiores aos parâmetros indicados, onde o grupo 20F:80S está 42,86\% acima, enquanto o tratamento 80F:20S obteve números $125,71 \%$ maiores que a descrição feita pelo NRC (2007).

Em outros estudos o aumento do consumo de matéria com uso de volumoso extrusado foi reportado. De acordo com Silva et al. (2020a) ao avaliarem os efeitos nutricionais provocados pela substituição de silagem de milho por ração extrusada em ovinos adultos, constataram CMS de $1,36 \mathrm{~kg} /$ dia para o tratamento contendo $20 \%$ de alimento volumoso extrusado e $80 \%$ de silagem de milho, e, 2,03 kg/dia no grupo que consumiu $80 \%$ de alimento extrusado e $20 \%$ de silagem de milho, reforçando que a substituição de silagem de milho por volumosos extrusados pode promover aumento no CMS.

Não houve efeito da substituição da silagem de milho pelo volumoso extrusado na digestibilidade da matéria seca $(\mathrm{P}>0,05)$ (Tabela 2). Embora o maior consumo de matéria seca possa ser um indicativo de redução da digestibilidade este fator não ocorreu com aumento na maior proporção de volumoso extrusado. A manutenção da digestibilidade é um indicativo de equilíbrio na fermentação ruminal e no aproveitamento do alimento, favorecendo a utilização do volumoso extrusado na alimentação de ruminantes.

Em relação à média, a digestibilidade da matéria seca foi de $56,95 \%$, sugerindo alto aproveitamento dos nutrientes, uma vez que o CMS de todos os grupos foi elevado. Valadares Filho (2018) descreveu que a DMS média da silagem de milho produzida no Brasil é de 59,58\%, dessa forma, a DMS verificada nesse estudo aproxima-se do valor encontrado para silagem, contribuindo para inferências de que é possível substituir a mesma pelo volumoso extrusado utilizado nesse experimento.

Não houve diferenças estatísticas $(\mathrm{P}>0,05)$ para as variáveis consumo de água $\left(\mathrm{CH}_{2} \mathrm{O}\right)$ e consumo de água em relação ao $\mathrm{CMS}\left(\mathrm{CH}_{2} \mathrm{O} / \mathrm{CMS}\right)$ (Tabela 3$)$. Chamamos a atenção que embora o volumoso extrusado seja um alimento seco (85\% de MS) em comparação a silagem de milho $(28,02 \%$ de MS) o consumo de água não foi um 
Silva, D. A. de P. et al.

limitante. Em relação ao recomendado para ovinos o NRC (2007) sugere que ovinos adultos, o consumo de água é de 2 a $3 \mathrm{~L} / \mathrm{kg}$ de matéria seca e a média obtida para os grupos avaliados na variável $\mathrm{CH}_{2} \mathrm{O} / \mathrm{CMS}$ foi de 2,04 $\mathrm{L} / \mathrm{kg}$, é possível inferir que a substituição do volumoso extrusado não afetou a ingestão hídrica das ovelhas.

Tabela 3 - Ingestão de água, parâmetros fecais e urinários de ovelhas consumindo níveis crescentes de Foragge ${ }^{\circledR} 70 \%$ em relação à silagem de milho

\begin{tabular}{|c|c|c|c|c|c|c|c|}
\hline & \multicolumn{4}{|c|}{ Tratamentos } & \multirow[b]{2}{*}{ P-valor } & \multirow[b]{2}{*}{ MG } & \multirow[b]{2}{*}{$\mathrm{CV}$} \\
\hline & $20 \mathrm{~F}: 80 \mathrm{~S}$ & 40F:60S & $60 \mathrm{~F}: 40 \mathrm{~S}$ & $80 \mathrm{~F}: 20 \mathrm{~S}$ & & & \\
\hline $\mathrm{CH}_{2} \mathrm{O}\left(\mathrm{L} . \mathrm{dia}^{-1}\right)$ & 3,24 & 3,73 & 4,52 & 3,97 & 0,4403 & 3,86 & 31,47 \\
\hline $\mathrm{CH}_{2} \mathrm{O} / \mathrm{CMS}(\mathrm{L} / \mathrm{kg})$ & 2,27 & 2,02 & 2,18 & 1,67 & 0,6856 & 2,04 & 31,44 \\
\hline VU (L. dia $\left.{ }^{-1}\right)$ & 1,4 & 1,59 & 1,17 & 1,08 & 0,8473 & 1,31 & 36,6 \\
\hline DU $\left(\mathrm{g} \cdot \mathrm{ml}^{-1}\right)$ & 1,0264 & 1,0222 & 1,0276 & 1,0339 & 0,3646 & 1,0275 & 0,99 \\
\hline PFMN (kg) & 2,14 & 2,44 & 3,67 & 3,32 & 0,0619 & 2,89 & 32,25 \\
\hline $\mathrm{PFMS}^{1}(\mathrm{~g})$ & 0,622 & 0,817 & 0,99 & 0,95 & 0,0486 & 0,845 & 24,28 \\
\hline MSF (\%) & 29,96 & 33,45 & 27,30 & 30,05 & 0,0511 & 30,19 & 10,41 \\
\hline $\mathrm{EF}^{*}$ & $2,08 \mathrm{~B}$ & $2,00 \mathrm{~B}$ & $2,68 \mathrm{~A}$ & $2,60 \mathrm{~A}$ & 0,0481 & 2,34 & - \\
\hline
\end{tabular}

CH2O: consumo de água; CMS: consumo de matéria seca; VU: volume de urina; DU: densidade da urina; PFMN: peso das fezes na matéria natural; PFMS: peso das fezes na matéria seca; MSF: matéria seca fecal; EF: escore fecal. *Estatística não paramétrica, utilizando teste de Kruskal e Walis. MG: média geral; CV: coeficiente de variação; ${ }^{1} \mathrm{Y}=0,556130+0,005786 \mathrm{x}, \mathrm{R}^{2}=80,95 \%$.

Com relação aos parâmetros urinários, também não houve diferenças entre os grupos avaliados $(\mathrm{P}>0,05)$ (P>0,05) (Tabela 3). A manutenção dos parâmetros urinários em relação aos tratamentos é reflexo a dependência dessas variáveis ao consumo de água. Em relação as recomendações para espécie, de acordo com Hendrix (2005), para ovinos, a recomendação de VU excretado por animal é de 0,1 a $0,4 \mathrm{~L}$ para cada $10 \mathrm{~kg}$ de peso vivo. Sendo assim, todos os tratamentos atingiram a normalidade, uma vez que a faixa ideal para os animais com peso de $54,5 \mathrm{~kg}$ seria de 0,545 a $2,18 \mathrm{~L} \mathrm{dia}^{-1}$, e obteve-se média de 1,31L para essa variável. Já a DU específica de ovinos, segundo Hendrix (2005), varia entre 1,020$1,040 \mathrm{~g} \mathrm{Ml}^{-1}$, portanto, não foram verificadas alterações na mesma uma vez que todos os grupos avaliados se apresentam inclusos nessa referência.

Para os parâmetros fecais foi observada resposta linear positiva para o peso das fezes na matéria seca (PFMS) $(\mathrm{P}<0,05)$ (Tabela 3). Esses resultados estão correlacionados com o CMS, uma vez que conforme aumentou a massa consumida pelos animais, sem alterar na digestibilidade, há maior produção de massa fecal. Avaliando os parâmetros fecais de ovelhas adultas consumindo volumoso extrusado em comparação à silagem de milho, Silva et al. (2020a) além de observar o aumento no consumo também constatou elevações nos níveis de PFMS.

Houve diferença estatística para o escore fecal (EF) $(\mathrm{P}<0,05)$ (Tabela 3). O tratamento com 60F:40S e 80F:20S foram superiores aos demais. De acordo com Ferreira et al. (2013) a presença de fibra na dieta deixa as fezes mais firmes, entretanto, o processo de extrusão promove a quebra da fibra presente nas paredes celulares dos alimentos (Reddy \& Reddy, 2015). Sendo assim, o aumento no EF nos tratamentos contendo maiores índices do volumoso extrusado pode ter sido devido à quebra da fibra durante a extrusão. Entretanto, essa diferença não afetou negativamente essa variável, uma vez que segundo Gomes et al. (2012) o EF considerado adequado é dois, similar ao apresentados em todos os tratamentos.

No comportamento ingestivo a substituição da silagem de milho por volumoso extrusado não alterou o tempo de alimentação, ócio e mastigação das ovelhas $(\mathrm{P}>0,05)$ (Tabela 4). No entanto, houve efeito quadrático para o tempo de ruminação $(\mathrm{P}<0,05)$ (Tabela 4).

O efeito quadrático no tempo gasto na ruminação foi influenciado pelo tratamento com maior inclusão de volumoso extrusado ofertado (80F:20S). Isto pois, ao aumentar a proporção de volumoso extrusado reduz o tamanho das partículas ingeridas, além de aumentar a facilmente de degradação pelos microrganismos ruminais. Esse fator colaborou para um menor gasto de tempo para que o alimento atinja tamanho suficiente e consiga seguir para o retículo. A redução do tempo de ruminação pode ser um indicativo de acidose ruminal (Antanaitis et al., 2019).

De acordo com Van Soest (1994), o tempo de ruminação médio diário para ovinos adultos é entre 240 a 540 minutos, sendo diretamente influenciado pela natureza da dieta e proporcional ao teor de parede celular dos alimentos volumosos. Todos os tratamentos atingiram a referência média de ruminação diária, onde o grupo que passou o menor tempo nesse processo foi o 80F:20S 
Avaliação da substituição da silagem de milho por volumoso extrusado na dieta de ovinos

(340 minutos) e o maior 40F:60S (505 minutos). Nesse sentido, é possível afirmar que mesmo diante de CMS elevados, o tempo gasto para ruminação esteve dentro do recomendado, não prejudicando a saúde dos animais.

Tabela 4 - Comportamento ingestivo de ovelhas recebendo Foragge ${ }^{\circledR} 70 \%$ em substituição à silagem de milho

\begin{tabular}{|c|c|c|c|c|c|c|c|}
\hline & \multicolumn{4}{|c|}{ Tratamentos } & \multirow[b]{2}{*}{ P-valor } & \multirow[b]{2}{*}{ MG } & \multirow[b]{2}{*}{ CV } \\
\hline & $20 \mathrm{~F}: 80 \mathrm{~S}$ & 40F:60S & 60F:40S & 80F:20S & & & \\
\hline ING (min) & 290 & 278 & 263 & 299 & 0,8022 & 282,50 & 24,41 \\
\hline RUM $^{1}$ (min) & 504 & 505 & 503 & 340 & 0,0239 & 463,00 & 19,48 \\
\hline ÓCIO (min) & 646 & 657 & 674 & 801 & 0,2012 & 694,50 & 17,61 \\
\hline MAST (min) & 794 & 783 & 766 & 639 & 0,2012 & 745,50 & 16,41 \\
\hline EING (g/min) & 5,37 & 6,97 & 7,91 & 8,28 & 0,0936 & 7,13 & \\
\hline $\mathrm{ERUM}^{2}(\mathrm{~g} / \mathrm{min})$ & 2,96 & 3,71 & 4,17 & 8,28 & 0,0143 & 4,78 & 30,98 \\
\hline EMAST $^{3}(\mathrm{~g} / \mathrm{min})$ & 1,98 & 2,40 & 2,71 & 4,04 & 0,0136 & 2,76 & 33,68 \\
\hline
\end{tabular}

ING: ingestão; RUM: ruminação; MAST: mastigação; E: eficiência; MG: média geral; CV: coeficiente de variação; ${ }^{1} \mathrm{Y}=381,50+7,780 \mathrm{x}-0,10250 \mathrm{x}^{2}$, $\mathrm{R}^{2}=93,81 \% ;{ }^{2} \mathrm{Y}=0,0684120+0,082034 \mathrm{x}, \mathrm{R}^{2}=78,84 \% ;{ }^{3} \mathrm{Y}=1,077040+0,033787 \mathrm{x}, \mathrm{R}^{2}=90,46 \%$

Com relação a eficiência das atividades no comportamento ingestivo, não houve diferença para a eficiência de ingestão (Eing) $(\mathrm{P}>0,05)$, porém houve efeito linear crescente para a eficiência de ruminação (Erum) e eficiência de mastigação (Emast) $(\mathrm{P}<0,05)$ (Tabela 4). $\mathrm{Na}$ nutrição de ruminantes, a utilização de alimentos volumosos é fundamental, uma vez que a fibra possui capacidade de aumentar a mastigação e ruminação. Dietas que não estimulam esses processos reduzem a produção de saliva e consequentemente, diminuem o $\mathrm{pH}$ ruminal e digestibilidade da fibra (Geron et al., 2013). Dessa forma, a variável ruminação pode ter influenciado na igualdade estatística constatada na DMS (Tabela 2), uma vez que o tratamento 80F:20S apresentou o maior consumo e a menor ruminação.
Já a melhora na eficiência de ruminação e mastigação é resultado da maior facilidade dos animais em apreender os alimentos, devido ao formato do Foragge ${ }^{\circledR}$ $70 \%$. É possível notar que os animais que receberam a distribuição $20 \mathrm{~F}: 80 \mathrm{~S}$ ingeriram $5,37 \mathrm{~g} / \mathrm{min}$, ao passo que o grupo contendo $80 \mathrm{~F}: 20 \mathrm{~S}$ ingeriu $8,28 \mathrm{~g} / \mathrm{min}$, cerca de $54,19 \%$ a mais de alimento por minuto, contribuindo para os CMS verificados de 1,50 e 2,37kg animal dia-1, respectivamente.

Ao avaliar os níveis glicêmico, houve resposta linear crescente em função do aumento da inclusão do Foragge ${ }^{\circledR} 70 \%$, entretanto, para suas concentrações por horário foi observado resposta linear decrescente $(\mathrm{P}<0,05)$ (Tabela 5).

Tabela 5 - Concentração glicêmica por tratamento e horário de coleta de ovinos alimentados com diferentes relações de Foragge ${ }^{\circledR} 70 \%$ e Silagem de milho

\begin{tabular}{|c|c|c|c|c|c|c|c|c|}
\hline & \multicolumn{5}{|c|}{ Tratamentos } & \multirow[b]{2}{*}{ P-valor } & \multirow[b]{2}{*}{ MG } & \multirow[b]{2}{*}{$\mathrm{CV}$} \\
\hline & \multicolumn{2}{|c|}{ 20F:80S } & 40F:60S & 60F:40S & $80 F: 20 S$ & & & \\
\hline Glicemia $^{1}$ (mg/Dl) & \multicolumn{2}{|c|}{44,24} & 51,84 & 49,20 & 55,08 & 0,0227 & 50,09 & 22,32 \\
\hline \multicolumn{6}{|c|}{ Horários de coleta } & \multirow[b]{2}{*}{$\mathrm{P}$} & \multirow[b]{2}{*}{ MG } & \multirow[b]{2}{*}{ CV } \\
\hline & $08 \mathrm{~h}$ & $11 \mathrm{~h}$ & $14 \mathrm{~h}$ & $17 \mathrm{~h}$ & $20 \mathrm{~h}$ & & & \\
\hline Glicemia $^{2}$ (mg/dL) & 51,60 & 55,20 & 52,30 & 45,05 & 46,35 & 0,0371 & 50,09 & 19,99 \\
\hline
\end{tabular}

MG: média geral; CV: coeficiente de variação; P: probabilidade de significância $(\mathrm{P}<0,05) .{ }^{1} \mathrm{Y}=42,620+0,14940 \mathrm{x}, \mathrm{R}^{2}=70,88 \%$; ${ }^{2} \mathrm{Y}=59,680$ $0,6350 x, R^{2}=58,25 \%$

Em ruminantes, cerca de $90 \%$ da glicose formada e utilizada são provenientes de gliconeogênese, processo onde o ácido graxo propionato é convertido nesse polímero (Kozloski, 2017). Como o produto extrusado promoveu um maior consumo pelos animais (Tabela 2) e possui maiores concentrações de nutrientes digestíveis totais e amido, com relação à silagem de milho (Tabela 1 ), esses fatores podem ter contribuído para a diferença verifica na variável glicemia por tratamento, promovendo utilização mais eficiente da glicose no metabolismo dos animais. O grupo 80F:20S apresentou o maior CMS e também a maior concentração de glicose por tratamento, reforçando que o consumo dos animais pode ter corroborado com tais índices. Mesmo com a diferença verificada, todos os tratamentos mantiveram-se inclusos 
na referência de glicose por Silva et al. (2020b) que é de 30-94 mg/dL para ovinos.

Com relação à redução nos níveis de glicose plasmática ao longo dia, esse acontecimento pode estar diretamente ligado ao manejo alimentar utilizado ao longo do dia de coleta glicêmica, já que o pico de glicose ocorreu às 11:00 horas (3:00 horas após o fornecimento da alimentação) e reduzido nas horas subsequentes por causa da degradação e fermentação da ração no ambiente ruminal. Esse processo demanda alto gasto energético e consequentemente, reduziu as concentrações de glicose ao longo do dia.
Já para os metabólitos sanguíneos, a variável triglicerídea obteve diferenças estatísticas para os tratamentos 20F:80S e 80F:20S, sendo que os demais tratamentos se apresentaram iguais, estatisticamente (Tabela 6). Os triglicerídeos correspondem à principal forma de armazenamento de ácidos graxos de cadeia longa (Kozloski, 2017). O tratamento que apresentou o maior nível de triglicerídeos continha $80 \%$ de silagem de milho, sendo este um alimento responsável por grande produção de ácido acético no rúmen, que é um dos ácidos graxos voláteis precursores da síntese de acetato. Entretanto, como as médias verificadas para todos os tratamentos enquadram-se na referência de Silva et al. (2020b), é possível inferir que houve adequado aporte energético as ovelhas.

Tabela 6 - Metabólitos energéticos e proteicos de ovelhas consumindo níveis crescentes de volumoso extrusado perante silagem de milho

\begin{tabular}{ccccccccc}
\hline & \multicolumn{9}{c}{ Tratamentos } & & & & \\
\cline { 2 - 7 } Metabólitos (mg/dL) & 20F:80S & 40F:60S & $\mathbf{6 0 F}: 40 S$ & $\mathbf{8 0 F}: 20 S$ & MG & P-valor & CV & VR* $^{*}$ \\
\hline Triglicerídeos & 28,00 & 18,30 & 19,90 & 16,40 & 20,65 & 0,0452 & - & $5-71$ \\
Colesterol & 68,50 & 62,80 & 58,70 & 67,40 & 64,35 & 0,8547 & 18,77 & $14-126$ \\
Ureia $^{1}$ & 16,50 & 19,60 & 24,40 & 20,70 & 20,50 & 0,0025 & 18,73 & $10-92$ \\
Proteínas Totais & 4,40 & 4,36 & 4,08 & 4,77 & 4,40 & 0,3741 & 19,26 & $3,1-10,7$ \\
Ácido úrico & 0,34 & 0,28 & 0,28 & 0,24 & 0,28 & 0,4587 & - & $0-1,7$ \\
Albumina & 3,68 & 3,43 & 3,44 & 3,30 & 3,46 & 0,2584 & 12,22 & $1,1-5,2$ \\
Creatinina & 0,83 & 0,80 & 0,78 & 0,77 & 0,79 & 0,3015 & - & $0,4-1,7$ \\
\hline
\end{tabular}

MG: média geral; CV: coeficiente de variação; VR*: Valor de referência segundo Silva et al. (2020b); ${ }^{1} \mathrm{Y}=15,950+0,087 \mathrm{x}, \mathrm{R}^{2}=47,45 \%$.

Já o colesterol que também faz parte do metabolismo energético, não apresentou diferenças (Tabela 6) $(P>0,05)$. Em ruminantes, o colesterol possui biossíntese no intestino delgado e células adiposas, sendo sintetizado a partir do acetil-CoA originado do ácido acético produzido no rúmen. É precursor dos ácidos biliares e dos hormônios esteroides, além de ser um indicador dos níveis de lipídeos na corrente sanguínea (Brito et al., 2016). Como seus níveis encontram-se na referência para ovinos de Silva et al. (2020b), é possível inferir que houve armazenamento adequado de energia e os níveis lipídicos não foram influenciados, durante o período experimental.

Com relação aos metabólitos proteicos, a ureia apresentou resposta linear positiva à inclusão de volumoso extrusado (Tabela 6) $(\mathrm{P}<0,05)$. Como um indicativo ao status proteico em ruminantes, já que apresenta relações diretas com a digestão proteica e metabolismo dos microrganismos do rúmen. Embora o volumoso extrusado utilizado tenha menor concentração de proteína, o processo gera a desnaturação das proteínas, fazendo com que sejam mais prontamente digeridas no rúmen (Reddy \& Reddy, 2015). Por isso, ao aumentar a proporção de volumoso extrusado na dieta houve resposta linear na concentração de ureia circulante nas ovelhas. Vale ressaltar que os índices obtidos para essa variável estão inclusos na referência de Silva et al. (2020b) para espécie.

As proteínas totais estão diretamente relacionadas com o status nutricional do animal já a albumina como ambos os metabólitos se encontram inclusos na referência de Silva et al. (2020b), a dieta ofertada contribuiu positivamente no metabolismo proteico das ovelhas avaliadas, indicando que elas possuíam aporte proteico normal e bom estado nutricional durante o ensaio experimental.

A creatinina é um importante indicativo da função renal dos animais, visto que é um metabólito que sofre pouca influência de fatores como dieta, idade ou sexo, além de refletir a filtração renal (Kozloski, 2017). A manutenção da concentração de creatinina circulante das ovelhas mesmo com a substituição em até $80 \%$ da silagem por volumoso extrusado indica não levar a problemas renais no curto prazo. Outro ponto positivo do uso do volumoso extrusado em até $80 \%$ foi a manutenção do $\mathrm{CH}_{2} \mathrm{O}$ e VU apresentaram-se adequados, e, os índices de creatinina estão inclusos no valor de referência de Silva et al. (2020b), é possível inferir que a função renal das 
Avaliação da substituição da silagem de milho por volumoso extrusado na dieta de ovinos

unidades experimentais não foi restringida ou prejudicada durante a realização desse experimento.

O ácido úrico possuí ligação estreita com o metabolismo ruminal recente, sendo influenciado pela qualidade nutricional do alimento ingerido e com a produção de proteína microbiana. A manutenção da concentração de ácido úrico ao substituir a silagem de milho por volumoso extrusado em até $80 \%$ indica que não houve mudanças em termos qualitativos da dieta e da produção de proteína microbiana (Tabela $60(\mathrm{P}>0,05)$. Como ele encontra-se na referência de Silva et al. (2020b), os tratamentos ofertados possuem ampla qualidade nutricional e não prejudicam a formação de proteína microbiana.

\section{Conclusão}

A substituição da silagem de milho pelo volumoso extrusado pode ser realizada em até $80 \%$ melhora o consumo, sem causar quaisquer prejuízos no equilíbrio ruminal, no comportamento ingestivo e no perfil metabólico a ovelhas adultas.

\section{Agradecimento}

Os autores agradecem a empresa Nuttrata ${ }^{\circledR} \mathrm{Nu}$ trição Animal, Itumbiara - GO, Brasil.

\section{Referências}

Antanaitis, R., Juozaitienė, V., Malašauskienė, D., \& Televičius, M. 2019. Can rumination time and some blood biochemical parameters be used as biomarkers for the diagnosis of subclinical acidosis and subclinical ketosis?. Veterinary and Animal Science, 8, 100077. Doi: https://doi. org/10.1016/j.vas.2019.100077.

Brito, D. R. B., Rocha, V. N. C., Cutrim Junior, J. A. A., Chaves, D. P., Silva, E. C. V., Coelho A. P., Soares, E. S., Silva E. M., \& silva, I. C. S. 2016. Perfil bioquímico de ovinos alimentados com níveis de inclusão do resíduo úmido de cervejaria. Revista brasileira de higiene e sanidade animal, 10 (4): 572-586. Doi: http://dx.doi.org/10.5935/1981-2965.20160048.

Conover, W. J. 1980. Practical Nonparametric Statistics. 2nd Edition, John Wiley \& Sons, New York.

Ferreira, S. F.; Guimarães, T. P.; Moreira, K. K. G.; Alves, V. A.; Lemos, B. J. M.; Souza, F. M. 2013. Caracterização fecal de bovinos. Revista Científica Eletrônica de Medicina Veterinária, Garça, 11 (20): 1-22.

Fischer, V.; Deswysen, A. G.; Dèspres, L.; Dutilleul, P.; Lobato, J. F. P. 1998. Padrões nectemerais do comportamento ingestivo de ovinos. Revista Brasileira de Zootecnia, 27: 362-369.

Geron, L. J. V., Mexia, A. A., Cristo, R. L., Garcia, J., Cabral, L. S., Trautmann, R. J., Martins, O. S., Zeoula, L. M. 2013. Consumo, digestibilidade dos nutrientes e características ruminais de cordeiros alimentados com níveis crescentes de concentrado em ambiente tropical no Vale do Alto Guaporé - MT. Semina: Ciências Agrária, 34 (5): 2497 2510. Doi: https://doi.org/10.5433/1679-0359.2013v34n5p2497.

Gomes, S. P; Borges, I.; Borges, A. L. C. C.; Macedo Junior, G. L.; Campos, W. E.; Brito, T. S. 2012. Tamanho de partícula do volumoso e frequência de alimentação sobre o metabolismo energético e proteico em ovinos, considerando dietas com elevada participação de concentrado. Revista Brasileira de Saúde e Produção Animal, 13: 732-744. Doi: https://doi. org/10.1590/S1519-99402012000300013.

Hendrix, C. M. 2005. Procedimentos laboratoriais para técnicos veterinários (4th ed.). São Paulo: Rocca, 556p.

Kaneko, J. J., Harvey, J. W., \& Bruss, M. L. 2008. Clinical biochemistry of domestic animals (6th ed.). San Diego: Academic Press, 928p.

Kozloski, G. V. 2017. Bioquímica dos ruminantes (3rd ed.). Rio Grande do Sul: editora UFSM, 203p.

Kruskal, W. H.; Wallis, W. A. 1952. Use of ranks in one-criterion variance analysis. Journal American Statistical Association. 47: 583-621. Doi: https://doi.org/10.1080/01621459.1952.10483441.
Macêdo, A. J. S.; César Neto, J. M.; Silva, M. A.; Santos, E. M. 2019. Potencialidades e limitações de plantas forrageiras para ensilagem: Revisão. Revista Brasileira de Higiene e Sanidade Animal, 13 (2): 320 - 337. Doi: https://doi.org/10.5935/1981-2965.20190024.

Maynard, D. G.; Stewart, J. W. B.; Bettany, J. R. 1984. Sulfur cycling in grassland and parkland soils. Biogeochemistry. 1: 97-111. Doi: https:// doi.org/10.1007/BF02181123.

Mourão, R. C.; Pancoti, C. G.; Moura, A. M.; Ferreira, A. L.; Borges, A. L. C. C.; Silva, R. R. 2012. Processamento do milho na alimentação de ruminantes. PUBVET, 6 (5): 1-27. Doi: https://doi.org/10.22256/ pubvet.v16n5.1292.

National Research Council - NRC. 2007. Nutrient Requeriments of Small Ruminants. 1. ed. Washington, DC, USA.

Oliveira, K. A., de Lima Macedo, G., da Silva, S. P., Araújo, C. M., Varanis, L. F. M., \& Sousa, L. F. 2018. Nutritional and metabolic parameters of sheep fed with extrused roughage in comparison with corn silage. Semina: Ciências Agrárias, 39(4), 1795-1804. http://dx.doi. org/10.5433/1679-0359.2018v39n4p1795.

Oliveira, K. A., Júnior, G. D. L. M., Araújo, C. M., Siqueira, M. T. S., de Araújo, M. J. P., de Jesus, T. A. V. 2019. Productive parameters of growing lambs fed an extruded ration with different roughage: concentrate ratios. Semina: Ciências Agrárias, 40(13), 3641-3652. http://dx.doi. org/10.5433/1679-0359.2019v40n6Supl3p3641.

Polli, V. A.; Restle, J.; Senna, D. B.; Rosa, C. E.; Aguirre, L. F.; Silva, J. H. S. 1996. Aspectos relativos à ruminação de bovinos e bubalinos em regime de confinamento. Revista Brasileira de Zootecnia, 25: 987-993. Doi: https://doi.org/10.1590/S0103-84781995000100024.

Reddy, G.V.N. \& Reddy, Y. R. 2015. Extrusion Technology. Animal Feed Technology, 32: 311-326.

SAS Institute Inc, JMP ${ }^{\circledR} 10$ Modeling and Multivariate Methods. 2012. Cary, NCSAS Institute Inc.

Silva, D. A. P., Santana, A. G., Araújo, C. M., Oliveira, K. A., Siqueira, M. T. S., Macedo Júnior, G. L. 2020a. Avaliação dos efeitos nutricionais e metabólicos da substituição de silagem de milho por ração extrusada de Capim-marandu (Urochloa brizantha) em ovinos. Caderno de ciências agrárias, 12: 01-09. https://doi.org/10.35699/2447-6218.2020.19833.

Silva, D. A. P., Varanis, L. F. M., Oliveira, K. A., Sousa, L. M., Siqueira, M. T. S., Macedo Júnior, G. L. 2020b. Parâmetros de metabólitos bioquímicos em ovinos criados no Brasil. Caderno de ciências agrárias, 12: 01-08. https://doi.org/10.35699/2447-6218.2020.20404. 
Silva, D. A. de P. et al.

Silva, E. N.; Duarte, J. B.; Reis, A. J. 2015. Seleção da matriz de variânciacovariância residual na análise de ensaios varietais com medidas repetidas em cana-de-açúcar. Ciência Rural 45: 993-999. Doi: http:// dx.doi.org/10.1590/0103-8478cr20141531.

Valadares Filho, S. C.; Lopes, S.A.; Machado, P.A.S.; Chizzotti, M.L; Amaral, H.F.; Magalhães, K.A.; Rocha Junior, V.R.; Capelle, E.R. 2018. CQBAL 4.0. Tabelas Brasileiras de Composição de Alimentos para Bovinos. Disponível em: www.ufv.br/cqbal. Acesso em: 10 de Janeiro de 2021.
Van Soest, P. J. 1994. Nutritional ecology of the ruminant. 2.ed. Ithaca: Cornell University Press. Doi: https://doi.org/10.7591/9781501732355.

Zereu, G. 2016. Factors Affecting Feed Intake and Its Regulation Mechanisms in Ruminants A Review. International Journal of Livestock Research, 6 (4): 1-22. Doi: https://doi.org/10.5455/ ijlr.20160328085909. 